\title{
Poverty: third wave behavioral approaches
}

This article will review the literature related to poverty and third wave behavioral approaches. It will specifically focus on Acceptance and Commitment Therapy (ACT) and the wider field of Contextual Behavioral Science (CBS). Having outlined CBS and poverty, this article will begin by highlighting evidence where people with psychological or physical health problems, who also live in poverty, are treated with ACT. It will then explore the possibility of tackling poverty itself and consider some limitations of traditional therapeutic approaches in this regard. Finally, it will review the potential for the CBS movement to deliver wider cultural change that reduces poverty itself. This article will speak to poverty that occurs in both the developed and developing world, although much of the evidence in relation to ACT and CBS is currently in the former.

Keywords: Acceptance and Commitment Therapy (ACT), Contextual Behavioral Science (CBS), Poverty.

Thompson, M. (2015). Poverty: third wave behavioral approaches. Current Opinion in Psychology, 2, 102-106. doi:10.1016/j.copsyc.2014.11.010

Article DOI: http://dx.doi.org/10.1016/j.copsyc.2014.11.010

NOTICE: this is the author's version of a work that was accepted for publication in Current Opinion in Psychology. Changes resulting from the publishing process, such as peer review, editing, corrections, structural formatting, and other quality control mechanisms may not be reflected in this document. Changes may have been made to this work since it was submitted for publication. A definitive version was subsequently published in Current Opinion in Psychology, 2, 102-106.

doi:10.1016/j.copsyc.2014.11.010

Posted following relevant equivalent guidelines: http://www.sherpa.ac.uk/romeo/issn/1471-4892/ 


\begin{abstract}
This article will review the literature related to poverty and third wave behavioral approaches. It will specifically focus on Acceptance and Commitment Therapy (ACT) and the wider field of Contextual Behavioral Science (CBS). Having outlined CBS and poverty, this article will begin by highlighting evidence where people with psychological or physical health problems, who also live in poverty, are treated with ACT. It will then explore the possibility of tackling poverty itself and consider some limitations of traditional therapeutic approaches in this regard. Finally, it will review the potential for the CBS movement to deliver wider cultural change that reduces poverty itself. This article will speak to poverty that occurs in both the developed and developing world, although much of the evidence in relation to ACT and CBS is currently in the former.

Keywords: Acceptance and Commitment Therapy (ACT), Contextual Behavioral Science (CBS), Poverty.
\end{abstract}




\section{Introduction}

This article will explore the literature related to poverty, Acceptance and Commitment Therapy (ACT) and Contextual Behavioral Science (CBS). In scope, the article will begin by exploring the evidence for conducting ACT with people who live in poverty. Then it will focus on tackling poverty itself, examining the potential of CBS in this regard. As this is the first review of this area, it will employ a relatively wide time frame - although focusing on more recent articles where available. To begin, first CBS and then poverty will be defined.

\section{Contextual Behavior Science (CBS)}

ACT is a third wave, contextual, therapy [1,2]. This review will not introduce ACT itself as the information is available elsewhere [3-5]. However it is important to note that ACT, along with the basic psychological science Relational Frame Theory (RFT) [6,7] is part of CBS, which has philosophical roots in "functional contextualism" [8-10]. Although ACT is arguably the most well known part of CBS, the wider mission of CBS is to: "create a behavioral science more adequate to the challenges of the human condition" [10]. This broad mission includes "psychopathology" and traditional psychotherapy and is also wider than this. For example, a recent article outlining the scope of CBS explicitly states that future research should focus on: "social disparities, environmental degradation, global climate change, poverty, child deprivation, and similar matters" [10]. This article will gather together the existing evidence in the area of poverty.

\section{Defining poverty}

Definitions of poverty vary. Research notes that an annual income of $\$ 1,095$ - \$2,190 was the poverty threshold for a family of three living in Africa, when an equivalent threshold of $\$ 14,680$ was in place for a family of three living in the US [11]. Similarly, while some benchmark poverty against 
absolute thresholds, other use relative values. More widely, while some argue that global poverty targets are being met [12], others suggest that this is partly because the targets for both poverty [13] and hunger [14] have been altered.

Moreover, even defining poverty based on income levels alone risks ignoring the increasingly studied role of income inequality [15-17] or other deprivations. Indeed, many working with global poverty now refer to "capabilities": asking what individuals are able to do and be, and what freedoms they have in areas like education and health [18]. Considerations like these are now included in measures such as the Human Development Index (HDI) and the Inequality-adjusted Human Development Index [19]. All of the above suggest that contemporary definitions of poverty tend to refer to a lack of resources or capability such that it impacts on an individuals participation in society, their health or well-being.

\section{ACT and poverty}

In the following section, examples of evidence for using ACT to work with people suffering from psychological or physical health difficulties, who also live in poverty will be highlighted. The evidence base for working clinically with those living in poverty is often bracketed under titles such as nondominant or under-represented groups, under-served or diverse populations and multicultural or sociocultural competency. Indeed the APA text, “Addressing cultural complexities in practice”, places poverty within an "S" of the "ADDRESSING" framework: Age, Developmental and acquired Disabilities, Religion, Ethnicity, Socioeconomic status, Sexual orientation, Indigenous heritage, National origin, and Gender [20].

In 2013, a special series focused on the use of third wave therapies for diverse populations was published. It contained 7 pieces [21-27]. Two pieces were commentaries and of the main four articles, none presented any new quantitative data. However, the introduction contained an updated meta analysis of studies treating diverse populations using third wave approaches, including data from 32 RCTs [23]. 
The meta analysis reported an overall effect size ranging from .38 to 1.32 (Hedges' g). The effect size of . 38 was found when comparing to treatment as usual, and 1.32 when comparing to waiting list. In terms of Hedges' g: .2 or above is small, .5 or above medium, and .8 or above a large effect size. Differences between Cohen's d and Hedges' g are limited, however Hedges' g may be better at correcting for bias from small samples. This is relevant as the median study size was $n=28$.

Naturally, not all the research from the meta analysis is relevant to this review. Some studies focused on other third wave approaches, or other populations. However relevant ACT research was present. For example, in terms of poverty in the developed world, one study worked with patients being treated for Type 2 diabetes in a low-income community health center in the US [28]. Patients were treated with either education, or education and ACT. Patients receiving ACT reported better self-care and other outcomes at three months (Hedges' g: .57).

The meta-analysis also highlighted poverty work in the developing world. Two RCTs compared ACT to other treatments for those with drug resistant epilepsy in South Africa [29] and India [30]. In both studies, ACT performed strongly (Hedges' g: $1.46 \&$.82). The South African study, included a dramatic reduction in the "seizure index" data collected in diary form, from 2156 at pre treatment, to 23 at post treatment, 34 at 6 month follow up and 55 at 1 year. Equally, as well as both studies taking place outside of the US or Europe, treatment was often delivered via an interpreter. Moreover, in the South African study, the report indicates that participants were living under the "minimum existence poverty level".

The studies above provide preliminary evidence that ACT can be helpful to those with health conditions who are also living in poverty in the US or globally. Other literature explores the use of ACT more generally with diverse or multicultural populations [31-33]. It is also worth noting that the Association for Contextual Behavioral Science (ACBS) has a Developing Nations Training Fund that supports professionals from developing nations to attend ACT conferences and to support ACT training to be held in those nations (http://contextualscience.org/developing_nations_fund). Similarly, psychologists 
POVERTY: THIRD WAVE BEHAVIORAL APPROACHES

are currently working with locals in Sierra Leone to deliver ACT based workshops through an organization called "commit and act" (www.commitandact.com/).

\section{The limits of therapy}

Providing psychological interventions for those living in poverty who also suffer from mental and physical health problems is vital. Evidence on the inequality of treatment provision reminds us how much work remains to be done in terms of both physical [34] and mental health [35]. However the next section of this review will explore both the limits and the possibility of using psychological science to attempt to reduce poverty itself.

The evidence that links poverty [36,37] and income inequality [15-17] to multiple other social, physical and psychological problems seems clear. Indeed researchers point to family and neighborhood poverty as being causal in the development of many of these problems in the developed world [36]. If this is the case, then the treatment of poverty and inequality itself seems to be important. However, some have noted that clinical psychology specifically and forms of individual and group therapy more generally can be limited in the face of such challenge [38-40]. Indeed the area of community psychology grew up partly in response to these concerns. Perhaps terminology from both community psychology and prevention science can help illustrate these limitations.

One distinction exists between micro, meso and macro levels of working [40]. In general, micro level work takes place with individuals; meso level work involves families, schools and workplaces; while macro level work embraces communities and wider society. Another pair of terms highlight differences between two levels of change: amelioration and transformation [40]. Put simply, amelioration refers to reducing the symptoms of the problem, although the fundamental problem remains. Whereas transformation changes the conditions that give rise to the problem in the first place. Typically transformation refers to change taking place at a community or societal level rather than within an 
individual alone. More recently, a third term related to levels of change has been introduced: co-optation [41]. This refers to the possibility that the work of some professionals, like psychologists, might become aligned, often unwittingly, with social forces that preserve the status quo [41].

Using these terms, perhaps you can see that individual therapy tends to happen at the micro level and be ameliorative in nature. However, while the terms themselves might be useful, this does not mean that community psychology necessarily has the answers or evidence in terms of other ways of working. Indeed, in a recent commentary it was noted that: "we [community psychologists] do mostly ameliorative work, we hope to do transformative work, and in some instances we even fall prey to co-optation" [41].

If the path to more transformative work is unclear to community psychology, it can be even less clear to clinical psychology [42]. In contrast to the above, the wider CBS literature is beginning to suggest that it has a framework that can not just improve the well-being of individuals living in poverty (micro / ameliorative), but can also change the wider culture that perpetuates poverty in the first place (meso, macro / transformative).

\section{The potential of CBS}

In two recent articles, Anthony Biglan, Dennis Embry and colleagues discuss the potential of CBS to influence cultural practices and bring about cultural change [43, 44]. By culture, they do not mean art, literature and music in isolation, but "everything that humans do" [43]. In this way, as discussed above, they are potentiality targeting macro levels of working and transformative levels of change. In one of these articles, entitled "a framework for intentional cultural change" the authors suggest that it may be possible for behavioral science to prevent many of the problems that affect human well-being [43]. It is noteworthy that the target for their interventions is not just individuals, but also organizations, policy and the media. So, for example, in the case of smoking, not just the behavior of individual smokers, but the behavior of the tobacco industry its marketing machine and wider public policy. Similar arguments are 
made, on a broader canvas, in "evolving the future: toward a science of intentional change" [44]. This paper includes concrete examples of how evidence based change has been advanced in community wide interventions. In both articles, similar to ACT informed therapeutic work, the usefulness of influencing psychological flexibility is highlighted. However so is the creation and promotion of more nurturing environments. Nurturing environments are those which help decrease the incidence of psychological problems by, among other things, reducing detrimental biological and psychological influences on behavior (toxic conditions) and increasing pro social behavior (prosociality) [43-45].

One concrete example is the "Promise Neighborhoods Research Consortium" (PNRC: http://promiseneighborhoods.org/ [37]). This group is promoting educational and developmental outcomes within high-poverty and distressed neighborhoods in the US. The PNRC seeks to influence family, school, peer, and wider neighborhood environments in an integrated fashion. The multifaceted approach consists of four work groups: 1. networking, 2. technology, 3. measurement and 4. intervention. The intervention group itself breaks down into different areas including: programs, policies and kernels (simple evidence based ways to influence behavior).

It is hopefully clear that coordinated interventions such as the PNRC have the potential to have a wider impact on reducing poverty, than individual therapy alone. However there is also an acknowledgment that even education, training and therapy combined might be limited in impact without considering the impact of organizational behavior on changing cultural practices $[46,47]$.

In this context, organizational behavior does not refer to employee well-being, but instead to the "negative externalities" that organizations can cause. A negative externality is a harm or cost imposed on an individual or community by a business or corporation. Obvious examples include pollution, or the long term effects of production and marketing of certain products by tobacco, alcohol and food industries [46]. The literature in this area also refers directly to poverty in the US and economic inequality $[46,47]$. One article notes evidence for a fall in poverty in elderly populations in the US in recent decades, but a 
corresponding rise in poverty for children and adults [46]. The article suggests that this rise is related to public policy that was less favorable to poorer families. Importantly, there is the suggestion that the policy change was influenced, in part, by business interests and the impact of their lobbying [46].

To counter negative externalities such as these, Biglan suggests the following four steps $[46,47]$. Firstly, to research which organizational behavior contributes to social problems. Secondly, to understand why the organizational behaviors persists. Thirdly, to assess what policies could influence the organization to stop and finally, to both study and develop successful advocacy organizations to lobby for the required change. The increasing restrictions on the tobacco industry provide an illustration of how this framework can be applied.

While developing advocacy programs may seem far removed from the more traditional work of the ACT therapist, it is important to note that this work is guided by the same framework: functional contextualism $[8,39,48]$. Also, while the above section tends to focus on poverty in the developed world, it is hopefully clear how a focus on organizational behavior could be applied to global poverty. The potentially negative influence of global business and global legal frameworks on developing nations is well documented [49,50]. While wider areas of psychology continue to produce research related to global poverty [51], it is possible that these researchers could utilize the frameworks highlighted above to compliment their ongoing work.

\section{Conclusion}

This article has reviewed the evidence base for the involvement of ACT and CBS in poverty. Evidence exists that supports the use of ACT for treating those with psychological or health difficulties who also live in poverty in different locations around the world. However, there also seems a clear need to address poverty itself. Traditional individual therapy, with a micro and ameliorative focus, may be limited in its ability to bring about this level of change. In contrast a wider CBS framework appears to 
have the potential to contribute to the reduction of poverty itself. Work is already applying ideas related to CBS, not just in the therapy room, but also in impoverished neighborhoods. In general this framework focuses on creating environmental contexts which contain more positive and less detrimental biological and psychological influences. As such, the focus of this work shifts for individual behavior in isolation to also include groups, neighborhoods, organizations and the influence of the media and public policy. The work may even necessitate increasing the influence of psychologically informed advocacy in order to reduce the negative externalities of organizations. With the above in mind, CBS appears to be making strides towards becoming a behavioral science more adequate to the challenges of the human condition. While further work and more time will be required to see whether the evidence supports these ideas: the potential for transformative change seems real. 
1. Hayes SC, Strosahl KD, Wilson KG: Acceptance and commitment therapy : An experiential approach to behavior change. Guilford Press; 1999.

2. Hayes SC, Strosahl KD, Wilson KG: Acceptance and commitment therapy: The process and practice of mindful change. Guilford Press; 2011.

3. Thompson M: ACT: a new acronym you need to know about. Clin. Psychol. Forum 2007, 172:1922.

4. Hayes SC, Luoma J, Bond F, Masuda A, Lillis J: Acceptance and commitment therapy: Model, processes, and outcomes. Behav. Res. Ther. 2006, 44:1-25.

5. Hayes SC, Levin ME, Plumb-Vilardaga J, Villatte JL, Pistorello J: Acceptance and commitment therapy and contextual behavioral science: Examining the progress of a distinctive model of behavioral and cognitive therapy. Behav. Ther. 2013, 44:180-198.

6. Hayes SC, Barnes-Holmes D, Roche B: Relational frame theory: A post-Skinnerian account of human language and cognition. Kluwer Academic/Plenum Publishers; 2001.

7. Dymond S, Roche B: Advances in relational frame theory: Research and application. New Harbinger; 2013.

8. Biglan A, Hayes SC: Should the behavioral sciences become more pragmatic? The case for functional contextualism in research on human behavior. Appl. Prev. Psychol. 1996, 5:47-57.

9. Fox EJ: Constructing a pragmatic science of learning and instruction with functional contextualism. Educ. Technol. Res. Dev. 2006, 54:5-36. 
* 10. Hayes SC, Barnes-Holmes D, Wilson KG: Contextual behavioral science: Creating a science more adequate to the challenge of the human condition. J. Context. Behav. Sci. 2012, 1:1-16.

Recent introduction to the area known as Contextual Behavioral Science (CBS).

11. Iceland J: Measuring poverty: Theoretical and empirical considerations. Meas. Interdiscip. Res. Perspect. 2005, 3:199-235.

12. United Nations: Millennium development goals report 2012. United Nations; 2012.

13. Reddy S G, Pooge T: How not to count the poor. Initiative for Policy Dialogue; 2009.

14. Lappé FM, Clapp J, Anderson M, Broad R, Messer E, Pogge T, Wise T: How we count hunger matters. Ethics Int. Aff. 2013, 27:251-259.

15. Wilkinson RG, Pickett KE: Income inequality and population health: A review and explanation of the evidence. Soc. Sci. Med. 2006, 62:1768-1784.

16. Wilkinson R, Pickett K: The spirit level: Why equality is better for everyone. Penguin; 2010.

17. Wilkinson RG, Pickett KE: The problems of relative deprivation: Why some societies do better than others. Soc. Sci. Med. 2007, 65:1965-1978.

18. Nussbaum MC: Capabilities as fundamental entitlements: Sen and social justice. Fem. Econ. 2003, 9:33-59.

19. United Nations Development Programme (UNDP): Human development report 2014 - Sustaining human progress: Reducing vulnerabilities and building resilience. United Nations Development Programme; 2014. 
20. Hays PA: Addressing cultural complexities in practice: Assessment, diagnosis, and therapy. American Psychological Association; 2008.

21. Dimidjian S, Kleiber B: Being mindful about the use of mindfulness in clinical contexts. Cogn. Behav. Pract. 2013, 20:57-59.

22. Dutton MA, Bermudez D, Matas A, Majid H, Myers NL: Mindfulness-based stress reduction for low-income, predominantly african american women with PTSD and a history of intimate partner violence. Cogn. Behav. Pract. 2013, 20:23-32.

* 23. Fuchs C, Lee JK, Roemer L, Orsillo SM: Using mindfulness- and acceptance-based treatments with clients from nondominant cultural and/or marginalized backgrounds: Clinical considerations, meta-analysis findings, and introduction to the special series. Cogn. Behav. Pract. 2013, 20:1-12.

Introduction to a wider special issue focusing on treating individuals from nondominant backgrounds with third wave approaches. Also details a meta-analysis of work that has been undertaken in this area.

24. Hinton DE, Pich V, Hofmann SG, Otto MW: Acceptance and mindfulness techniques as applied to refugee and ethnic minority populations with PTSD: Examples from "culturally adapted CBT." Cogn. Behav. Pract. 2013, 20:33-46.

25. La Roche M, Lustig K: Being mindful about the assessment of culture: A cultural analysis of culturally adapted acceptance-based behavior therapy approaches. Cogn. Behav. Pract. 2013, 20:60-63.

26. Petkus AJ, Wetherell JL: Acceptance and commitment therapy with older adults: Rationale and 
POVERTY: THIRD WAVE BEHAVIORAL APPROACHES

considerations. Cogn. Behav. Pract. 2013, 20:47-56.

27. Sobczak LR, West LM: Clinical considerations in using mindfulness- and acceptance-based approaches with diverse populations: Addressing challenges in service delivery in diverse community settings. Cogn. Behav. Pract. 2013, 20:13-22.

28. Gregg JA, Callaghan GM, Hayes SC, Glenn-Lawson JL: Improving diabetes self-management through acceptance, mindfulness, and values: A randomized controlled trial. J. Consult. Clin. Psychol. 2007, 75:336-343.

29. Lundgren T, Dahl J, Melin L, Kies B: Evaluation of acceptance and commitment therapy for drug refractory epilepsy: A randomized controlled trial in South Africa - a pilot study. Epilepsia 2006, 47:2173-2179.

30. Lundgren T, Dahl J, Yardi N, Melin L: Acceptance and commitment therapy and yoga for drugrefractory epilepsy: A randomized controlled trial. Epilepsy Behav. EB 2008, 13:102-108.

31. Woidneck MR, Pratt KM, Gundy JM, Nelson CR, Twohig MP: Exploring cultural competence in acceptance and commitment therapy outcomes. Prof. Psychol. Res. Pract. 2012, 43:227-233.

32. Hayes SC, Muto T, Masuda A: Seeking cultural competence from the ground up. Clin. Psychol. Sci. Pract. 2011, 18:232-237.

33. Masuda A: Mindfulness and acceptance in multicultural competency: A contextual approach to sociocultural diversity in theory and practice. New Harbinger Publications; 2014.

34. Thompson M: Bias, prejudice, and disparities in health care. In Mindfulness and Acceptance in Behavioral Medicine: Current Theory and Practice. Edited by McCracken LM. New Harbinger; 
2011:269-302.

35. Collins PY, Patel V, Joestl SS, March D, Insel TR, Daar AS, Bordin IA, Costello EJ, Durkin M, Fairburn C, et al.: Grand challenges in global mental health. Nature 2011, 475:27-30.

36. Yoshikawa H, Aber JL, Beardslee WR: The effects of poverty on the mental, emotional, and behavioral health of children and youth: implications for prevention. Am. Psychol. 2012, 67:272284.

37. Komro KA, Flay BR, Biglan A, Promise Neighborhoods Research Consortium: Creating nurturing environments: A science-based framework for promoting child health and development within high-poverty neighborhoods. Clin. Child Fam. Psychol. Rev. 2011, 14:111-134.

38. Albee G: The futility of psychotherapy. J. Mind Behav. 1990, 11:369-384.

39. Biglan A, Hinds E: Evolving prosocial and sustainable neighborhoods and communities. Annu. Rev. Clin. Psychol. 2009, 5:169-196.

40. Prilleltensky I, Nelson G: Community psychology: Reclaiming social justice. In Critical psychology: An introduction. Edited by Fox D, Prilleltensky I. Sage Publications; 1997:166-184.

41. Prilleltensky I: Meaning-making, mattering, and thriving in community psychology: From cooptation to amelioration and transformation. Psychosoc. Interv. 2014, 23:151-154.

42. Thompson M: Exploring the trainees' view of a socio-political approach within UK clinical psychology. J. Community Appl. Soc. Psychol. 2007, 17:67-83.

* 43. Biglan A, Embry DD: A framework for intentional cultural change. J. Context. Behav. Sci. 2013, 2:95-104. 
Introduces a pragmatic, contextual, framework of cultural change. Amongst other things, the paper highlights the importance of prosociality, minimizing toxic events, creating nurturing environments and increasing psychological flexibility.

* 44. Wilson DS, Hayes SC, Biglan A, Embry DD: Evolving the future: Toward a science of intentional change. Behav. Brain Sci. 2014, 37:395-416.

Extends the literature on potential cultural change. Includes evidence of change at small and large population levels.

45. Biglan A, Flay BR, Embry DD, Sandler IN: The critical role of nurturing environments for promoting human well-being. Am. Psychol. 2012, 67:257-271.

* 46. Biglan A: Corporate externalities: A challenge to the further success of prevention science. Prev. Sci. Off. J. Soc. Prev. Res. 2011, 12:1-11.

Highlights the important role of corporate externalities, including in the area of poverty. Presents a framework to help counter these influences.

47. Biglan A: The role of advocacy organizations in reducing negative externalities. J. Organ. Behav. Manag. 2009, 29:215-230.

48. Biglan A: Changing cultural practices: A contextualist framework for intervention research. Context Press; 1995.

49. Barkemeyer R, Holt D, Preuss L, Tsang S: What happened to the "development" in sustainable development? Business guidelines two decades after Brundtland. Sustain. Dev. 2014, 22:15-32.

50. Hulme D, Scott J: Governing development: Power, poverty and policy. In Governing the world? 
Cases in global governance. Edited by Harman S, Williams D. Routledge; 2013:28-45.

51. Carr SC, Thompson M, Dalal AK, de Guzman JM, Gloss A, Munns L, Steadman A: Psychology and poverty reduction: A global special issue. Int. Perspect. Psychol. Res. Pract. Consult. 2014, 3:215237. 